\title{
Estilos de aprendizaje y minería de datos: un estudio preliminar en el contexto universitario
}

\section{Learning styles and data mining: a preliminary study in the university context}

\author{
Cruz Vergaraz ${ }^{1}$, Ana Oviedo²,Claudia Carmona³ ,Gloria Vélez4, Iván Amón5. \\ Recibido para publicación: 14 de septiembre 2018 - Aceptado para publicación: 10 de octubre 2018
}

\section{RESUMEN}

Este articulo presenta hallazgos preliminares de la investigación en curso sobre estilos de aprendizaje y rendimiento académico en estudiantes de ingeniería de la Universidad Pontificia Bolivariana; para ello se ha hecho uso de la minería de datos como técnica para el ordenamiento y análisis de datos. La investigación se articula a la apuesta de la Universidad por prevenir la deserción estudiantil y mejorar las practicas pedagógicas de los docentes universitarios, teniendo como marco de referencia la apuesta del Ministerio de Educación Nacional (MEN-Colombia) de fortalecer las ciudades inteligentes.

En la aproximación teórica inicial, se identificó como instrumento principal para evaluar estilos de aprendizaje el cuestionario (CHAEA) de Honey y Alonso, con este cuestionario se inicia la recopilación de datos que posteriormente serán analizados con ayuda de diferentes algoritmos aplicados en analítica de datos.

Esta fase inicial de la investigación permitió evidenciar que la Minería de datos ha sido usada con frecuencia en estudios relacionados con rendimiento académico y deserción universitaria, en especial la técnica de árbol de problemas; no obstante, resulta de interés resaltar que aunque hay estudios sobre estilos de aprendizaje y minería de datos, no se encuentran estudios que ofrezcan a los docentes estrategias pedagógicas para mejorar su práctica en el aula, en su mayoría los estudios se quedan en una fase descriptiva.

PALABRAS CLAVE: Ciencia de Datos, Educación en una ciudad inteligente, Estilos de aprendizaje, Fracaso Escolar, Perfilamiento de Estudiantes.

\footnotetext{
${ }^{1}$ Licenciada en Educación Especial (Tecnológico de Antioquia, Medellín, Antioquia, Colombia 1997), Psicóloga con énfasis en Psicología Social (Universidad Católica Luis Amigó, Medellín, Antioquia, Colombia, 2007), Especialista en Docencia Investigativa Universitaria (Universidad Católica Luis Amigó, Medellín, Antioquia, Colombia, 2008) Magister en Psicología (Universidad Pontificia Bolivariana, Medellín, Antioquia, Colombia, 2013).

2 Ingeniera de Sistemas (Universidad de Antioquia, Medellín, Antioquia, Colombia, 2004), Magister en Ingeniería (Universidad de Antioquia, Medellín, Antioquia, Colombia, 2008) y Doctora en Ingeniería Electrónica (Universidad de Antioquia, Medellín, Antioquia, Colombia, 2013).

${ }^{3}$ Ingeniera Electrónica (Universidad Pontificia Bolivariana, Medellín, Antioquia, Colombia, 2001), Especialista en Telecomunicaciones (Universidad Pontificia Bolivariana, Medellín, Antioquia, Colombia, 2010) y Magíster en Ingeniería de Telecomunicaciones (Universidad de Antioquia, Medellín, Antioquia, Colombia, 2011).

${ }^{4}$ Ingeniera de Sistemas de la Universidad EAFIT, Magister en Gestión Tecnológica de la Universidad Pontificia Bolivariana y Doctora en Ingeniería Electrónica de la Universidad de Antioquia

${ }^{5}$ Ingeniero de Sistemas (Universidad Eafit, Medellín, Antioquia, Colombia, 1986), Especialista en Técnicas Computarizadas de Producción (Universidad Eafit, Medellín, Antioquia, Colombia, 2000)y Mágister en Ingeniería de Sistemas (Universidad Nacional, Medellín, Antioquia, Colombia, 2010).
} 


\section{ABSTRACT}

This article presents the preliminary findings of ongoing research on learning styles and academic performance in engineering students of the Universidad Pontificia Bolivariana. For the development of the research, data mining has been used as a technique for ordering and analyzing data.

The research is articulated with the commitment of the University to prevent student desertion and improve the pedagogical practices of university teachers, taking as a frame of reference the commitment of the Ministry of National Education (MEN-Colombia) to strengthen smart cities.

In the initial theoretical approach, the questionnaire (CHAEA) of Honey and Alonso was identified as the main instrument for evaluating learning styles. With the CHAEA questionnaire, the collection of data is started, which will later be analyzed with the help of different algorithms applied in data analytics.

This initial phase of the investigation made it possible to show that data mining has been used frequently in studies related to academic performance and university desertion, especially the problem tree technique: It is important to note that although there are studies on learning styles and data mining, there are no studies that offer teachers pedagogical strategies to improve their practice in the classroom, most studies are left in a descriptive phase.

KEY WORDS: Data Science, Education in a Smart City, Learning Styles, School Failure, Student Profiling.

\section{INTRODUCCIÓN}

En una ciudad inteligente, los ciudadanos deben contar con una educación inclusiva en torno a la diversidad cultural de sus habitantes. En pro de esta visión, las instituciones educativas deben propender por la formación integral de la población reconociendo la diversidad en el proceso de aprendizaje. Sin embargo, este objetivo se ve obstaculizado por diversas situaciones, en Colombia el $46.1 \%$ de los estudiantes universitarios no culminan sus estudios profesionales por diversos motivos: problemas personales, insuficiencia económica, bajo desempeño, entre otros (Ministerio de Educación Nacional, 2015).

La deserción estudiantil, es un tema que ha tomado especial atención en los últimos años. La deserción de estudiantes universitarios está vinculado al desempeño académico, a los procesos de selección y al rendimiento académico que logra el estudiante. El nivel de deserción es el resultado de la combinación y efecto de distintas variables (Eckert \& Suénaga, 2015)

Uno de los factores principales que conducen al abandono de la carrera universitaria es el bajo desempeño académico, que, aunque puede atribuirse al estudiante, también es responsabilidad de los docentes. Dicha responsabilidad puede derivarse del uso de metodologías de enseñanza que no se ajustan a las necesidades particulares de los estudiantes. En (Ortiz, López, Machado, \& Frutos, 2014), los autores plantean que una de las competencias necesarias de un docente es diseñar cursos acorde con las habilidades de aprendizaje de los estudiantes, para lo cual debe tener presente: condiciones de estudio, administración de su tiempo, hábitos y métodos, metas de aprendizaje y estrategias de aprendizaje.

Las instituciones de Educación Superior comúnmente cuentan con docentes profesionales con alta calidad académica, sin embargo, es frecuente encontrar que el desarrollo de los contenidos en las aulas no se lleve a cabo teniendo en la cuenta los diversos estilos de aprendizaje que caracteriza a los estudiantes, lo que posiblemente conlleva a elevar el porcentaje de fracaso escolar y por ende de deserción.

Estos hechos motivan el trabajo en conjunto de docentes de ingeniería y psicología entorno a la "Educación". Se formula entonces la siguiente pregunta "¿será posible a partir de la minería de datos evaluar estilos de aprendizaje de los estudiantes, y a partir de los resultados diseñar estrategias pedagógicas que permitan reducir el fracaso escolar y por ende la deserción estudiantil?".

Para afrontar esta situación se propone desarrollar una plataforma de ciencia de datos que permita (1) identificar los estilos de aprendizaje que revelan los estudiantes por medio de la aplicación de instrumentos, (2) determinar los perfiles 
de los estudiantes para diseños didácticos y metodologías de enseñanza más adecuados y (3) utilizar un modelo analítico que permitan predecir el fracaso escolar.

El proyecto se enfoca en el desarrollo de una plataforma que sirva de herramienta para que tanto los docentes como el personal administrativo de una institución educativa, puedan reconocer las fortalezas de los estudiantes y potenciarlas dentro del proceso de formación. A partir de los resultados se espera: 1) Estructurar perfiles de estudiantes, en lo que se describan los modos particulares de asimilar los aprendizajes; 2) Diseñar estrategias de enseñanza que se articulen a los estilos de aprendizaje de los estudiantes; 3) Minimizar el riesgo de fracaso escolar y de deserción, a partir de la implementación de una plataforma que permita la identificación de factores de riesgo.

El presente trabajo da cuenta de los avances del proyecto realizados hasta el momento en aspectos como el uso investigativo de los estilos de aprendizaje en la sección 2 y el análisis de técnicas de minería aplicadas a estudios similares en la sección 3. Posteriormente se presenta la metodología propuesta y los resultados obtenidos hasta el momento.

\section{USO INVESTIGATIVO DE LOS ESTILOS DE APRENDIZAJE}

En (SEGARRA, ESTRADA, \& MONFERRER, 2015) se dio a conocer los estilos de aprendizaje de los estudiantes universitarios de programas en distintos ámbitos (ciencias sociales y humanas, ciencias experimentales y ciencias económicas), para ello utilizaron el modelo del cerebro total para ajustar los métodos de enseñanza a los estilos de aprendizaje de los alumnos. En la investigación profundizaron en la comprensión del estilo de aprendizaje utilizado por los estudiantes universitarios, así como en el diseño de fórmulas óptimas para su mejora y el consecuente incremento de sus rendimientos académicos. A partir de las puntuaciones obtenidas para cada cuadrante, identificaron que, en general, los alumnos con mejor rendimiento académico son aquellos que tienen un perfil de dominancia mixto, es decir, que en su forma de pensar interconectan ambos hemisferios cerebrales.

En (Gargallo-López, Pérez-Pérez, Verde-Peleato, \& García-Félix, 2017), se presenta el impacto de los métodos centrados en el aprendizaje, implementados por cuatro profesores/as, en los estilos de aprendizaje de los alumnos. También se pretendía analizar la valoración del alumnado sobre los métodos utilizados. La muestra fue de 117 estudiantes, que cursaban estos estudios durante los años 2014-2015, repartidos en cuatro grupos de cuatro especialidades diferentes. Los profesores utilizaron métodos centrados en el aprendizajecon diferentes formatos metodológicos en los cuatro grupos. Se constataron mejoras significativas en un número importante de las variables que analiza el cuestionario (aprendizaje profundo, autorregulación, orientación centrada en el interés personal, uso del conocimiento, cooperación, etc) en los cuatro grupos, siendo las diferencias mayores en los grupos 1 y 4 que en los grupos 2 y 3 . Las comparaciones entre los grupos reflejaron diferencias significativas en el pretest entre los cuatro grupos, que no se dieron en el postest, igualándose los grupos. Los alumnos valoraron positivamente los métodos utilizados. Los resultados corroboraron la influencia positiva de los métodos centrados en los estilos de aprendizaje de los estudiantes, y aportan ideas para la mejora de los procesos de enseñanzaaprendizaje del alumnado universitario.

En (Aguilera Pupo \& Ortiz Torres, 2009) se señala que el concepto de estilos de aprendizaje es muy utilizado en las investigaciones debido a su valor teórico y metodológico para comprender y perfeccionar el proceso de enseñanza-aprendizaje en los diferentes niveles educativos por los que transitan los alumnos. Sin embargo, alrededor de este término existen disímiles interpretaciones de acuerdo con las bases teóricas que asume cada investigador. Precisamente, el objetivo del artículo es ofrecer una valoración sobre los antecedentes en las investigaciones sobre estilos de aprendizaje y las tendencias actuales de sus modelos explicativos, como recurso para favorecer la utilización de estrategias personalizadas de aprendizaje. Al interior del trabajo se hace una descripción de los principales instrumentos utilizados en el contexto educativo para evaluar estilos de aprendizaje; entre los que sobresale el modelo para clasificar los estilos de aprendizaje que propuso Silverman, F en 1998 (Aguilera Pupo \& Ortiz Torres, 2009); este modelo establece cinco dimensiones a partir del procesamiento de la información:

1) Por el canal sensorial que utiliza el alumno para ingresar la información: visuales y verbales.

2) De acuerdo con la fuente de información preferida: sensitivos e intuitivos. 
3) Según la organización de la información: inductivos y deductivos.

4) Por el progreso del procesamiento de la información: secuenciales y globales.

5) Por forma del procesamiento de la información: activos y reflexivos

A lo largo de la investigación se hace referencia a la articulación de aspectos cognitivos, psicológicos, intrínsecos y extrínsecos al estudiante, que tienen incidencia en la presencia del estilo de aprendizaje que lo caracteriza; siendo éste un factor esencial a tener en cuenta en una intervención psicopedagógica.

En Medellín, en la institución de educación superior Politécnico Jaime Isaza Cadavid, a partir de la aplicación inicial del Cuestionario Honey-Alonso de estilos de aprendizaje (CHAEA) a estudiantes de Licenciatura, se obtuvo un instrumento adaptado a las condiciones particulares de los estudiantes en este contexto. La adaptación se hizo para minimizar los conflictos de comprensión de algunos ítems, que fueron reportados por los estudiantes y para facilitar el uso del tiempo, ya que se pasó de un instructivo de 80 ítems a uno de 40. Conservar estas características del modelo CHAEA ha sido el marco de actuación en todo el proceso de adaptación del cuestionario a estudiantes antioqueños matriculados en el Politécnico Jaime Isaza, que alberga alumnos de todas las regiones del Departamento de Antioquia, e inclusive de otras regiones tales como la Costa Atlántica, el Centro y Suroccidente del país (Madrigal \& Trujillo, 2014). El cuestionario adaptado se reconoce como CAMEA 40. Teniendo en cuenta las anteriores consideraciones se ha adoptado el cuestionario CHAEA, para evaluar los estilos de aprendizaje en los estudiantes de ingeniería de la Universidad Pontificia Bolivariana, posteriormente se aplicará a los demás estudiantes, una vez se apruebe la plataforma por parte de los responsables.

\section{APLICACIONES DE MINERÍA DE DATOS PARA EL PERFILAMIENTO DE ESTUDIANTE}

En la búsqueda de las organizaciones por utilizar mejor la información que tienen de sus clientes y de sus procesos se han utilizado técnicas de ciencia de datos para muchos tipos de aplicaciones, una de ellas es el caso de la Universidad Simón Bolivar (Eckert \& Suénaga, 2015).

Las instituciones educativas no han sido ajenas al proceso y han comenzado a usar dichas técnicas en diferentes trabajos descritos a continuación.

Se encontró proceso de análisis enfocados en los estilos de aprendizaje (Núñez Cardenas, Hernández Palacios, Tomás Mariano, \& Felipe Redondo, 2013)(Fallis, 2013)(ALMENDRALES, 2016), para esto se utilizaron técnicas como minería de datos del tipo descriptiva (clustering) y el algoritmo utilizado es K-means (Núñez Cardenas et al., 2013), algoritmos de clasificación (Fallis, 2013)técnicas de reglas y árbol de decisión (Fallis, 2013) (ALMENDRALES, 2016) y análisis de correlaciones, regresión logística, PCA y análisis de componentes principales.(ALMENDRALES, 2016) con las cuales se logró definir el estilo de aprendizaje, factores que influían en el fracaso escolar y predecir el rendimiento académico.

Adicionalmente se encontraron análisis enfocados en el ambiente escolar(Brault, Janosz, \& Archambault, 2014), la participación escolar a través de redes sociales (Barragán Moreno \& González Támara, 2017) y en los espacios de participación definidos por la institución educativa (Wang \& Eccles, 2013) para estos análisis se usaron técnicas de teoría de grafos y Árbol de decisión(Barragán Moreno \& González Támara, 2017) y análisis multidimensional (SEM) (Wang \& Eccles, 2013) y análisis multinivel (Brault et al., 2014). Como resultado de dichos análisis se encontró que aplicación de la modelación matemática y la modelación estadística al estudio del uso de las redes sociales y de los mecanismos de comunicación fortalecen el conocimiento de la población estudiantil y de las dinámicas sociales de los grupos y se logra predicen cambios en los patrones de participación escolar

\section{MÉTODO}

Para abordar el proyecto se utiliza la metodología CRISP-DM(Chapman et al., 2000) la cual es usada para proyectos de analítica de datos, esta consta de 6 fases:

En la Fase 1 - comprensión del negocio, se establece que el proyecto es desarrollado en la Universidad Pontificia Bolivariana, donde interesados por el fracaso escolar, se desarrolla este proyecto de prevención.

En la Fase 2 - comprensión de los datos, se analizan los datos recolectados por la Universidad a través de los diferentes programas de apoyo a los estudiantes y las orientaciones de cada uno de los programas. Se revisan las variables 
necesarias para el proyecto mediante un rastreo bibliográfico y se evalúan de forma teórica diferentes instrumentos para la recolección.

En la Fase 3 - preparación de los datos, se ha encontrado que las fuentes de datos están dispersas, así que es necesario realizar una consolidación y una evaluación de la calidad de las variables. Dentro de este proceso se ha evidenciado que la Universidad ha modificado los instrumentos de recolección de información a través del tiempo, pero no se analizó el grado de correlación o la importancia de las variables descartadas.

En la Fase 4 - modelamiento, esta fase propende desarrollar tres tipos de modelos como apoyo a las labores administrativas, acompañamiento a estudiantes, y como herramienta a los docentes para la planeación de estrategias del curso. Los modelos diseñados son:

1) Análisis de los estilos de aprendizaje que revelan los estudiantes por medio de la aplicación de instrumentos como CHAEA y KOLB que permitan diseños didácticos y metodologías de enseñanza más adecuados. (Madrigal \& Trujillo, 2014)

2) Determinar los perfiles de los estudiantes mediante un análisis de clustering con el algoritmo k-means.

3) Crear un modelo analítico que permitan predecir el fracaso escolar mediante la técnica de árboles de decisión que permita identificar las variables más relevantes para dicha predicción.

En la Fase 5 - evaluación, se identifica el desempeño de los modelos, indicando medidas de calidad.

Finalmente, en la Fase 6 - despliegue, se estructuran los modelos en una plataforma para que puedan ser utilizados por docentes y administrativos.

\section{HALLAZGOS TEMPORALES}

Se puede concluir que el recurso educativo Existen distintos modelos de evaluación de estilos de aprendizaje, los cuales se derivan de propuestas psicológicas que han sido adoptadas por la educación. Entre las perspectivas de punta sobresalen: modelos cognitivos basados en el funcionamiento cerebral (teoría de los cuadrantes hemisféricos), modelos neuropsicológicos (plantean estos estilos: auditivo, visual, quinestésico) y modelos funcionales, basados en la percepción de los estudiantes sobre su modo de aprender. En este último, la propuesta de KOLB, y el cuestionario CHAEA, se ajusta a lo que se pretende con la investigación propuesta para UPB. En esta perspectiva los estilos de aprendizaje se clasifican en: activo, reflexivo, teórico y pragmático.

Existen investigaciones actuales sobre el uso de la minería de datos en evaluación de estilos de aprendizaje, en las que se visualiza su pertinencia en propuestas de mejoramiento de la educación. Asimismo, se encuentra en varias investigaciones que con el uso de minería de datos se pueden identificar factores de riesgo que se convierten en predictores de fracaso y deserción educativa.

Se puede extraer la técnica de árbol de decisión como una de las más utilizadas, acompañadas de otro tipo de experimentos para clasificar diferentes tipos de aspectos que resultan convenientes analizar a la luz de mejorar la calidad de la educación

La creación de plataformas de ciencia de datos, a partir de la minería se articula a la apuesta del MEN- en fomentar la educación por medio de las TIC, con el propósito de fortalecer la existencia de ciudades inteligentes, en las que sea posible una educativa inclusiva en la que se acoja la diversidad estudiantil.

Aun no se encuentran antecedentes importantes relacionados con el lugar del docente en la implementación de estrategias didácticas y metodológicas en el aula, que permitan articular estilos de aprendizaje con estilos de enseñanza, y poder así, plantear acciones que favorezcan la permanencia de los estudiantes en el contexto universitario.

\section{REFERENCIAS}

[1]. Aguilera Pupo, E., \& Ortiz Torres, E. (2009). Las investigaciones sobre los estilos de aprendizaje y sus modelos explicativos. Revista Estilos de Aprendizaje, 4(4), 1-19. Retrieved from http://learningstyles.uvu. edu/index.php/jls/article/view/167/125

[2]. Aguilera Pupo, E., \& Ortiz Torres, E. (2009). Las investigaciones sobre los estilos de aprendizaje y sus modelos explicativos. Revista Estilos de Aprendizaje, 4(4), 1-19. Retrieved from http://learningstyles.uvu. edu/index.php/jls/article/view/167/125

[3]. ALMENDRALES, G. (2016). Estudio sobre 
los estilos de aprendizaje mediante minería de datos como apoyo a la gestión directiva de la Institución Educativa Joaquín Cárdenas Gómez del municipio de SAN CARLOS. Medellín.

[4]. Barragán Moreno, S. P., \& González Támara, L. (2017). Approach to student dropouts from the perspective of social and academic integration. Revista de La Educacion Superior, 46(183), 63-86. https:// doi.org/10.1016/j.resu.2017.05.004

[5]. Brault, M. C., Janosz, M., \& Archambault, I. (2014). Effects of school composition and school climate on teacher expectations of students: A multilevel analysis. Teaching and Teacher Education, 44, 148-159. https://doi.org/10.1016/j.tate.2014.08.008

[6]. Chapman, P., Clinton, J., Kerber, R., Khabaza, T., Reinartz, T., Shearer, C., \& Wirth, R. (2000). Crisp-Dm 1.0. CRISP-DM Consortium, 76. https://doi.org/10.1109/ ICETET.2008.239

[7]. Eckert, K. B., \& Suénaga, R. (2015). Análisis de deserción-permanencia de estudiantes universitarios utilizando técnica de clasificación en minería de datos. Formacion Universitaria, 8(5), 3-12. https://doi. org/10.4067/S0718-50062015000500002

[8]. Fallis, A. . (2013). Encuentro Internacional de Educación en Ingeniería ACOFI 2014. Journal of Chemical Information and Modeling (Vol. 53). https://doi.org/10.1017/ CBO9781107415324.004

[9]. Gargallo-López, B., Pérez-Pérez, C., Verde-Peleato, I., \& García-Félix, E. (2017). Estilos de aprendizaje en estudiantes universitarios y enseñanza centrada en el aprendizaje. RELIEVE - Revista Electrónica de Investigación y Evaluación Educativa, 23(2), 1-24. https://doi.org/10.7203/ RELIEVE.23.2.9078

[10]. Madrigal, A. D. J., \& Trujillo, J. M. (2014). Adaptación del cuestionario Honey-Alonso de estilos de aprendizaje para estudiantes de una institución universitaria de Medellín, Colombia. Journal of Learning Styles, 7(13), 155-181. Retrieved from http:// learningstyles.uvu.edu/index.php/jls/article/ view/38

[11]. Ministerio de Educación Nacional. (2015). Estadísticas Deserción y Graduación 2015. Ministerio de Educación Nacional, 4. Retrieved from www.mineducacion. gov.co/sistemasdeinformacion/1735/ articles-357549_recurso_3.pdf

[12]. Núñez Cardenas, F. de J., Hernández Palacios, R., Tomás Mariano, V. T., \& Felipe Redondo, A. M. (2013). Identificación de Estilos de Aprendizaje en Alumnos Universitarios de Computación de la Huasteca Hidalguense mediante Técnicas de Minería de Datos, 1-7.

[13]. Ortiz, Y. G., López, D., Machado, D. C., \& Frutos, O. R. (2014). Rendimiento Academico 1. Edumecentro, 6(2), 272-277. Retrieved from http://www.revedumecentro. sld.cu

[14]. SEGARRA, M., ESTRADA, M., \& MONFERRER, D. (2015). Estilos de aprendizaje en estudiantes universitarios: lateralización vs. interconexión de los hemisferioscerebrales. (Spanish). University Students' Learning Styles: Lateralisation vs. Interconnection of Cerebral Hemispheres. (English), 73(262), 583-600. Retrieved from http://pbidi.unam.mx:8080/login?url=http:// search.ebscohost.com/login.aspx?direct=t rue $\& d b=z b h \& A N=110262208 \&$ lang $=$ es $\&$ sit e=eds-live

[15]. Wang, M. Te, \& Eccles, J. S. (2013). School context, achievement motivation, and academic engagement: A longitudinal study of school engagement using a multidimensional perspective. Learning and Instruction, 28, 12-23. https://doi. org/10.1016/j.learninstruc.2013.04.002 